\section{Spectral Finite Element Analysis of Random SHRINKAGE IN CONCRETE}

By Zdeněk P. Bažant, ${ }^{1}$ F. ASCE and Tong-Sheng Wang ${ }^{2}$

Agstract: The spectral method, previously generalized for aging linear systems, is applied in conjunction with the finite element method to analyze shrinkage stresses in aging viscoelastic structures exposed to random environmental humidity. The age-dependence of both drying diffusivity and creep properties are taken into account. The solution of pore humidity is obtained from a matrix differential equation in time, with complex-valued matrices. Elastic shrinkage stresses are then obtained from the matrix equations of the finite element method, in which the matrices are also complex-valued. The stresses in presence of aging creep are determined by a superposition integral in time based on the relaxation function. Numerical examples concerning a long cylindrical vessel exposed at the outer surface are given. The standard deviations of pore humidity and of stresses significantly vary with time, and their standard deviation exhibits fluctuations about a drifting mean. The solution is practically meaningful only if concrete does not crack, e.g., when a prestress sufficient to prevent cracking is introduced. For environmental fluctuations of long periods, such as one year, the computation is quite efficient; however, if shorttime fluctuations are considered, the computing time becomes very large.

\section{INTRODUCTION}

Shrinkage and creep of concrete exhibit greater random variability than any other mechanical property of concrete. Clearly, a probabilistic design approach which takes into account not only the mean effects of shrinkage and creep, but also their variance, must be developed in order to improve long-term serviceability and, for structures such as nuclear reactors, safety as well. One major factor causing random variability is the random fluctuation of the environment, particularly its relative humidity and temperature. This random fluctuation produces random shrinkage stresses and thermal stresses, which are significantly reduced by creep.

Under the assumption of linearity of all governing equations, the problems of shrinkage stresses and thermal stresses can be treated separately and, at the end of analysis, superimposed. Mathematically, both problems lead to the same type of equations, and so only the problem of shrinkage stresses will be studied in detail here. For the sake of simplicity, the random variability of material properties will be neglected, i.e., the constitutive law will be considered as deterministic

When the material does not age, shrinkage stresses as well as thermal stresses caused by a random stationary environment represent a stationary random process in time. Solution of a typical problem of this kind, concerned with random thermal stresses in an infinitely long cy-

'Prof. of Civ. Engrg. and Dir., Center for Concrete and Geomaterials, The Technological Inst., Northwestern Univ., Evanston, Ill. 60201.

${ }^{2}$ Visiting Scholar, Northwestern Univ.; on leave from the Huai River Commission, Bangbu, Anhui, China.

Note.-Discussion open until February 1, 1985. To extend the closing date one month, a written request must be filed with the ASCE Manager of Technical and Professional Publications. The manuscript for this paper was submitted for review and possible publication on September 7,1983 . This paper is part of the Joumal of Structural Engineering, Vol. 110, No. 9, September, 1984. CASCE, ISSN Joumal of Structural Engineering, Vol. 110, No. 9,
$0733-9445 / 84 / 0009-2196 / \$ 01.00$. Paper No. 19146. lindrical elastic vessel, was pioneered by Heller with co-workers (1620,23) who applied the spectral method (method of power response spectra). His analytical solution is, however, inapplicable to concrete, because the drying diffusivity and creep strongly depend on age. This causes the response to be a nonstationary random process in time. A solution of this problem for an aging viscoelastic vessel (e.g., a nuclear containment) was obtained in Ref. 24, in which the method of impulse response functions was used and only creep, but not the drying diffusivity, was considered to exhibit aging. The solution was analytic, based on Bessel and Kelvin functions.

The method of impulse response functions (24) is, however, computationally inefficient. Even though, in contrast to nonaging structures, the frequency response function of an aging structure must be determined by solving differential or integral equations in time, the spectral densities of input and response remain related algebraically, the same as for nonaging structures $(5,11)$. On the other hand, the autocorrelation functions of the input and the response, on which the impulse response function method is based, are related by integrals. Another reason for the efficacy of the spectral approach is the fact that the environment can usually be well described by only a few periodic components.

Therefore, the spectral method was generalized for aging systems $(5,6)$, and its application was demonstrated for shrinkage stresses in an aging viscoelastic halfspace (11). The last formulation was, however, limited in its solution of the spatial problem, which was carried out for a halfspace by numerical integration of certain explicit integrals. This approach is not possible in general, and the objective of the present study is to combine the spectral approach to aging linear systems with a finite element solution in space. This will obviously extend the applicability of the spectral approach to any concrete structure.

\section{Spectral Determination of Variance}

For a nonaging structure subjected to a stationary random environment (or loading), the statistical characteristics of the response at a given point within the structure are those of the random time variation of the response at that point. This concept is, however, inapplicable to an aging structure. In that case we must imagine an ensemble of a great number of identical structures exposed to different realizations of the same environment, to which each structure is exposed at the same age, $t_{0}$ $(5,6)$. The statistical characteristics of the response at certain age, $t$, and location, $x$, are then those of the ensemble of the response values for all these structures at age $t$ and location $x$. Actual calculation of the responses for all structures in this ensemble would, however, be prohibitively tedious. In view of the ergodic property of the environment, one may consider a single structure instead of an ensemble of many structures, and imagine that this structure is exposed to the same random environment at various times, provided that the age, $t_{0}$, at the beginning of exposure is the same for all cases. This means that we imagine the random environmental history to be shifted in time relative to the instant the structure is built $(5,6)$, and analyze the statistical properties as a function of the time shift. 
Let $\theta$ denote time (the actual time) measured, e.g., from the Creation or the Big Bang, and let $\tau$ be the time when the concrete was cast. Then $t=\theta-\tau$ represents the age of concrete. Determination of the statistical characteristics of the response now requires finding the response, $g\left(\mathbf{x}, t, t_{0}, \tau\right)$, and considering its random variation as a function of the shift, $\tau$, at fixed current age, $t$, fixed $t_{0}$, and fixed location, $x$. A rigorous derivation of the spectral approach based on this concept was given in Refs. 5-6. We will now indicate a simplified argument which suffices for determining the relationship between the variances of input and response.

In the spectral approach, the response to each periodic component of the environmental spectrum is calculated separately, and the responses for all periodic components are then superimposed. We may, therefore, restrict attention to a single periodic component of the environment (input), $f(t)=s_{0} e^{i \omega t}$, in which $\omega$ is the circular frequency (a real number); and $s_{0}$ may be regarded as the standard deviation (a real number). By an appropriate method of structural creep analysis, one can find the response, $g\left(x_{k}, t, t_{0}\right)$, at a location defined by Cartesian coordinates $x_{k}(k=$ $1,2,3)$. Introducing the notation $Y\left(\omega, x_{k}, t, t_{0}\right)=e^{-i \omega t} g\left(x_{k}, t, t_{0}\right) / s_{0}$, in which $Y(\ldots)$ is a complex function called the frequency response function, the response (a complex-valued function) may be expressed as

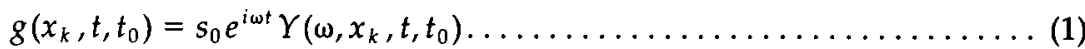

As argued before, determination of statistical characteristics of the response requires considering the environmental history as a function of the actual time, $\theta=t+\tau$, rather than concrete age, $t$. Thus, we consider the environmental history $s_{0} e^{i \omega \theta}$ which can be also written as $s_{0} e^{i \omega t} e^{i \omega \tau}$, and according to Eq. 1 the response is $s_{0} e^{i \omega \theta} Y\left(\omega, x_{k}, t, t_{0}\right) e^{i \omega \tau}$. Now the variance of the response may be calculated as the variance of all response values for all possible time shifts $\tau$ at fixed $t, t_{0}$, and $x_{k}$ :

$s_{0_{g}}^{2}=\operatorname{Var}_{\tau}\left[s_{0} e^{i \omega t} Y\left(\omega, x_{k}, t, t_{0}\right) e^{i \omega \tau}\right] \ldots \ldots \ldots \ldots \ldots \ldots \ldots \ldots \ldots \ldots \ldots \ldots \ldots$

$=E_{\tau}\left[s_{0} e^{i \omega t} Y\left(\omega, x_{k}, t, t_{0}\right) e^{i \omega \tau} s_{0} e^{-i \omega t} Y^{*}\left(\omega, x_{k}, t, t_{0}\right) e^{-i \omega t}\right] \ldots \ldots \ldots \ldots$

$=s_{0}^{2}\left|Y\left(\omega, x_{k}, t, t_{0}\right)\right|^{2}$

in which $E_{\tau}$ denotes the expectation based on averaging over all $\tau$; and $Y^{*}$ is the complex conjugate of $Y$. The standard deviation of the response is

$s_{0 g}=s_{0}\left|Y\left(\omega, x_{k}, t, t_{0}\right)\right|$

The environmental history may be approximately characterized as a superposition of several periodic components of frequencies, $\omega_{i}$, and standard deviations, $s_{0(j)} ; j=1,2, \ldots N$. Then the standard deviations of the given environment and of the response are given as

$s_{0}=\left(\sum_{j=1}^{N} s_{0(j)}^{2}\right)^{1 / 2} ; s_{0 g}=\left(\sum_{j=1}^{N} s_{0 g(j)}^{2}\right)^{1 / 2}$

$=\left[\sum_{j=1}^{N} s_{0(j)}^{2}\left|Y\left(\omega_{j}, x_{k}, t, t_{0}\right)\right|^{2}\right]^{1 / 2}$
Eq. 4 results from the relation

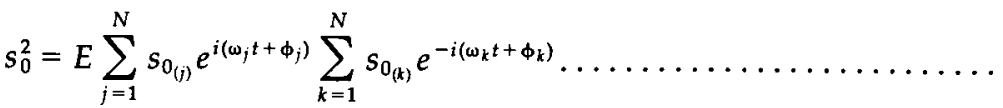

$$
\begin{aligned}
& s_{0}^{2}=E \sum_{j} \sum_{k} s_{0_{(j)}} s_{0_{(k)}} e^{i\left(\omega_{j}-\omega_{k}\right) t} e^{i\left(\phi_{j}-\phi_{k}\right)}=\sum_{j} s_{0_{(j)}}^{2}
\end{aligned}
$$

(in which $\phi_{j}=$ phase angles) if one uses the fact that $E\left(e^{i \omega t}\right)=0$ if $\omega \neq$ 0 ; and 1 if $\omega=0$. Alternatively, Eq. 4 may be obtained from relations $g(t)=g_{1}(t)+g_{2}(t)+\ldots+g_{N}(t)$ and

$$
\begin{aligned}
R_{g 8}\left(t_{1}, t_{2}\right)= & E\left\{\left[g_{1}\left(t_{1}\right)+g_{2}\left(t_{2}\right)+\ldots\right]\left[g_{1}\left(t_{2}\right)+g_{2}\left(t_{2}\right)+\ldots\right]\right\} \ldots \ldots \ldots \\
R_{g 8}\left(t_{1}, t_{2}\right)= & R_{11}\left(t_{1}, t_{2}\right)+R_{22}\left(t_{1}, t_{2}\right)+\ldots+R_{N N}\left(t_{1}, t_{2}\right) \\
& +R_{12}\left(t_{1}, t_{2}\right)+R_{21}\left(t_{1}, t_{2}\right)+\ldots R_{j k}\left(t_{1}, t_{2}\right)+\ldots \ldots \ldots \ldots \\
R_{g 8}\left(t_{1}, t_{2}\right)= & R_{11}\left(t_{1}, t_{2}\right)+R_{22}\left(t_{1}, t_{2}\right)+\ldots+R_{N N}\left(t_{1}, t_{2}\right) \ldots \ldots \ldots \ldots \\
s_{0 g}^{2}=R_{g g}(t, t)= & \sum_{j=1}^{N} R_{j j}(t, t)=\sum_{j=1}^{N} s_{0 g(j)}^{2} \ldots \ldots \ldots \ldots \ldots \ldots
\end{aligned}
$$

in which $R_{j i}=$ the autocorrelation function; and $R_{j k}=$ the cross-correlation functions.

In earlier works $(5,6)$, it was shown that an equation similar to Eq. 2 holds in general for the spectral densities, $S_{f}$ and $S_{g}$, of the environment (input) and the response

$S_{g}\left(\omega, \mathbf{x}, t, t_{0}\right)=S_{f}(\omega)\left|Y\left(\omega, \mathbf{x}, t, t_{0}\right)\right|^{2}$.

Function $S_{g}$ completely defines the second-moment statistical characteristics of the response.

The spectral approach reduces the stochastic problem to the determination of the complex-valued frequency response function $Y(\ldots)$. This subject occupies us next.

\section{Finite Element Analysis of Frequency Response for Humidity}

Drying of concrete may be described by a diffusion equation, which is known to be nonlinear $(3,9,12)$. However, the spectral analysis is feasible only for linear systems, and so this equation must be linearized. This is acceptable as an approximation if the value of drying diffusivity, $C$, is selected as the mean value of diffusivity for the anticipated range of humidity variation. Neglecting the direct effect of hydration on pore humidity $h$, the diffusion equation for concrete may be written as (9)

$\frac{\partial h}{\partial t}=C(t) \nabla^{2} h$

in which $\nabla^{2}$ is the Laplacian; $h$ is the relative vapor pressure in the pores of concrete; and $C(t)$ is the diffusivity. The value of diffusivity strongly depends on the age, $t$, of concrete, as experimentally demonstrated by Wierig (25) and others. 
Eq. 8 must further be supplemented by initial conditions, consisting in specified values of $h$ at $t=t_{0}$, and the boundary conditions, consisting in either specified values of $h$ (exposed surface), or a zero gradient of $h$ in the normal direction (sealed surface).

For finite element analysis, one introduces the column matrix $\mathbf{h}=\left(h_{1}\right.$, $\left.h_{2}, \ldots h_{N}\right)^{T}$ of the nodal values of $h(T=$ transpose $)$. Within individual finite elements, $h$ is approximated as $h(\mathbf{x}, t)=\mathbf{N}(\mathbf{x}) \mathbf{h}(t)$, in which $\mathbf{x}=$ coordinate vector; and $\mathbf{N}(\mathbf{x})=$ real-valued matrix of given shape functions of the finite element (distribution functions). Introducing this expression for $h$ into Eq. 8, writing for Eq. 8 the Galerkin variational equation, and applying Green's integral theorem, one then obtains the following matrix equation:

$\mathbf{K}_{1} \frac{\partial \mathbf{h}}{\partial t}+C(t) \mathbf{K}_{2} \mathbf{h}=0$

in which $\mathbf{K}_{1}$ and $\mathbf{K}_{2}=(N \times N)$ square matrices given as

$\mathbf{K}_{1}=\sum_{e l} \int_{e l} \mathbf{N}^{T} \mathbf{N} d A, \quad \mathbf{K}_{2}=\sum_{e l} \int_{e l} \mathbf{N}_{i,}^{T} \mathbf{N}_{, i} d A$.

Here "el" refers to integration over the element area, $A$, and to the summation over all finite elements; the subscript preceded by a comma denotes partial differentiation; and repetition of subscripts implies summation. Matrices $\mathbf{K}_{1}$ and $\mathbf{K}_{2}$ are real but $h$ is complex-valued.

Matrices $K_{1}$ and $K_{2}$ are set up without regard to the boundary conditions. Subsequently, the boundary conditions of prescribed surface humidity, $e^{i \omega t}$, are introduced by replacing in Eq. 9 the rows for the exposed surface nodes with the relations $h_{b}=e^{i \omega t}$ in which $h_{b}=$ humidity at exposed surface nodes. To implement these boundary conditions, it suffices to multiply those diagonal terms of matrix $\mathbf{K}_{2}$ which correspond to the exposed surface nodes by some very large number, e.g., $10^{30}$, and to replace Eq. 9 by the matrix equation

$\mathbf{K}_{1} \frac{\partial \mathbf{h}}{\partial t}+C(t) \overline{\mathbf{K}}_{2} \mathbf{h}=\mathbf{b} e^{i \omega t}$.

in which matrix $\overline{\mathbf{K}}_{2}$ results from $\mathbf{K}_{2}$ as described; and $\mathbf{b}=\mathbf{a}$ column matrix in which those elements which correspond to the exposed surface nodes are $10^{30}$, and all remaining ones are zero. Eq. 11 represents a system of $N$ nonhomogeneous first-order linear differential equations in time

It is now convenient to set $h\left(x_{k}, t\right)=H\left(\omega, x_{k}, t, t_{0}\right) e^{i \omega t}$ in which $H(\ldots)$ represents the frequency response function of pore humidity. Thus, the column matrix of nodal $h$-values may be expressed as

$\mathbf{h}=\mathbf{H}\left(\omega, x_{k}, t, t_{0}\right) e^{i \omega t}$

in which $\mathbf{H}(\ldots)$ is the column matrix of frequency response functions for pore humidities in the nodes (complex-valued). Substitution of Eq 12 into Eq. 11 yields the following equation for the evolution of $\mathbf{H}$ :

$\mathbf{K}_{1} \frac{\partial \mathbf{H}}{\partial t}+\left[C(t) \overline{\mathbf{K}}_{2}+i \omega \mathbf{K}_{1}\right] \mathbf{H}=\mathbf{b}$
Because of the dependence of diffusivity $C$ on time, the solution needs to be carried out numerically, in time steps $\Delta t$. The most efficient method for this purpose is the Crank-Nicolson algorithm, for which the values of $\mathbf{H}$ at time $t_{r+1}$ are solved from the following linear matrix equation:

$\mathbf{K}_{a} \mathbf{H}_{r+1}=\mathbf{K}_{b} \mathbf{H}_{r}+\mathbf{b}$

in which subscript $r$ refers to discrete time, $t_{r}$, and

$\mathbf{K}_{a}=\frac{1}{2}\left[C\left(t_{r+1 / 2}\right) \overline{\mathbf{K}}_{2}+i \omega \mathbf{K}_{1}\right]+\frac{1}{\Delta t} \mathbf{K}_{1}$,

$\mathbf{K}_{b}=\frac{1}{2}\left[C\left(t_{r+1 / 2}\right) \overline{\mathbf{K}}_{2}+i \omega \mathbf{K}_{1}\right]-\frac{1}{\Delta t} \mathbf{K}$

Repeated solutions of Eq. 15 yield the discrete values of the response to a periodic surface humidity of zero mean.

In general, the surface humidity variation consists of a deterministic part, $h$, and a periodic (or stochastic) part, $h$. The deterministic part describing the time variation of the mean surface humidity, produces deterministic response $\hat{h}\left(x_{k}, t, t_{0}\right)$. This deterministic solution must be superimposed on the solution for the periodic surface humidity determined as described previously for the boundary conditions of the type $\tilde{h}=s_{0} e^{i \omega_{0}\left(t-t_{0}\right)}$.

\section{Finite Element Analysis of frequency Response of Stress}

Pore humidity changes cause local shrinkage strains, $\epsilon_{s h}(x, t) . \mathrm{Al}$ though the dependence of $\epsilon_{s h}$ on $h$ is nonlinear (3), it needs to be linearized to make an analytical solution feasible. This linearization is characterized by a constant shrinkage coefficient, $\kappa_{s h}$ (a real number), representing the mean slope of the curve of $\epsilon_{s h}$ versus $h$ over the region of interest. Thus, $\boldsymbol{\epsilon}_{s h}(\mathbf{x}, t)=\kappa_{s h}[h(\mathbf{x}, t)-1]$. Since, however, we assume for the purpose of calculating the frequency response function that the initial value of $h$ is 0 rather than 1 , we use

$\epsilon_{s h}(\mathbf{x}, t)=\kappa_{s h} h(x, t)$

The shrinkage coefficient depends on age, $t$. However, this dependence is not strong and has been neglected in numerical calculations, although the same type of solution would be possible even if the dependence of $\kappa_{s h}$ on $t$ were considered.

Since the shrinkage strains are generally incompatible, they produce shrinkage stresses. These are strongly reduced by creep. The shrinkage stresses may be conveniently calculated on the basis of McHenry's analogy $(3,21)$. First, one obtains the elastic solution of shrinkage stresses, upon which the solution in the presence of creep may be obtained from a history integral of the elastic shrinkage stresses involving the relaxation function of concrete $(3,4)$

As is well known, the elastic solution may be obtained, according to the finite element method, from the matrix equation

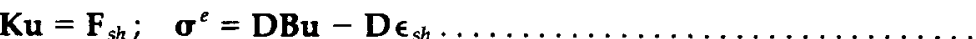


in which $\mathbf{u}$ is the column matrix of nodal displacements at any time, $t_{r}$; $D$ is the matrix of elastic constants of concrete based on Young's elastic modulus, $E_{c}$, at age 28 days; $\mathbf{B}$ is the geometric matrix; $\mathbf{K}$ is the structural stiffness matrix; and

$\mathbf{F}_{s h}=\sum_{e l} \int_{e l} \mathbf{B}^{T} \mathbf{D} \epsilon_{s h} d A ; \quad \boldsymbol{\epsilon}_{s h}=\left(\boldsymbol{\epsilon}_{s h}, \epsilon_{s h}, \boldsymbol{\epsilon}_{s h}, 0,0,0\right)^{T}$

in which $\boldsymbol{\epsilon}_{s h}=$ the column matrix of shrinkage strains at time $t_{r}$; and $\boldsymbol{\sigma}^{e}$ $=$ the matrix of elastic stresses. Note that matrices $\boldsymbol{\sigma}^{e}, \mathbf{u}, \boldsymbol{\epsilon}_{s h}$, and $\mathbf{F}_{s h}$ are complex-valued, while $\mathbf{B}, \mathbf{D}$, and $\mathbf{K}$ are real. Matrix $\mathbf{F}_{s h}$ represents the matrix of nodal forces equivalent to shrinkage strains. For fluctuating humidity given by Eq. 12, the shrinkage strain variation is in the form $\boldsymbol{\epsilon}_{s h}=\kappa_{s h} H\left(\omega, x_{k}, t, t_{0}\right) e^{i \omega t}$. Solving Eqs. 17 successively for all discrete times, $t_{r}(r=1,2,3 \ldots)$, one obtains the elastic stress column matrices $\boldsymbol{\sigma}_{(r)}^{e}$ for all discrete times, $t_{r}$

According to McHenry's analogy $(3,21)$, the stresses in presence of creep may be calculated as

$\boldsymbol{\sigma}(t)=\int_{t_{0}}^{t} \frac{R\left(t, t^{\prime}\right)}{E\left(t^{\prime}\right)} d \boldsymbol{\sigma}^{e}\left(t^{\prime}\right)$

in which $R\left(t, t^{\prime}\right)$ is the relaxation function of concrete, representing the stress at age $t$ caused by a unit constant strain imposed at age $t^{\prime}$. The relaxation function may be easily determined from any given compliance function, $J\left(t, t^{\prime}\right)$, either numerically, to a high degree of accuracy, or, somewhat less accurately, from an approximate algebraic formula (4). In numerical step-by-step analysis considered here, the integral in Eq. 19 may be approximated by the sum

$\boldsymbol{\sigma}_{(r)}=\frac{1}{E_{c}} \sum_{s=1}^{r-1} R\left(t_{s}, t_{s+1 / 2}\right)\left(\boldsymbol{\sigma}_{(s+1)}^{e}-\mathbf{\sigma}_{(s)}^{e}\right)$

in which the subscript $s+1 / 2$ refers to the middle of the time interval $\left(t_{s}, t_{s+1}\right)$. These sums must be evaluated for each stress component at each finite element of the structure in order to obtain the complete solution. When periodic surface humidity is used in the input, the solution $\boldsymbol{\sigma}_{(r)}$ represents the frequency response function of stress.

Due to the presence of periodic components, the time step must not exceed approximately $1 / 16$ of the shortest period involved. This could be quite demanding for computer time, since in the present approach it is not possible to choose time intervals, $\Delta t=t_{s+1}-t_{s}$, increasing with time in a geometric progression, as is usual in creep structural analysis for steady loads. However, it is possible to develop a different algorithm which permits the time step to be increased and reach values much larger than the fluctuation period (10).

\section{Application to Cylindaical Vessel}

Although the finite element aspect of the present solution is routine, we should indicate the detailed form of the matrices involved so that the subsequent example, concerning a cylindrical vessel, could be re-
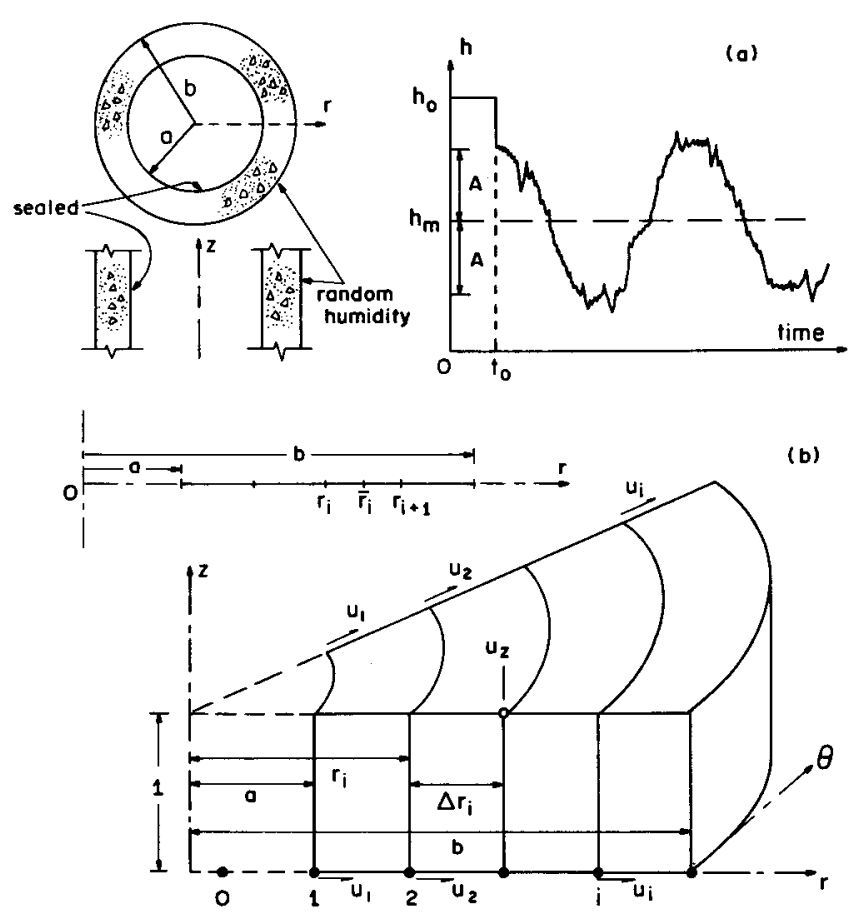

FIG. 1.-(a) Cylindrical Vessel and Random Variation of Surface Humidity $h$; and (b) One-Dimensional Discretization of Cylindrical Wall

produced and checked. We now consider a homogeneous, infinitely long cylindrical vessel (Fig. 1). The random surface humidity is prescribed on the outer surface and is the same everywhere. The internal surface is sealed by a steel liner whose stiffness is negligible.

The finite elements consist of concentric rings between the nodal points of radius coordinates $r_{i}$ [Fig. 1(b)]. The shape functions for the diffusion problem, $\mathbf{N}$, are chosen as linear in the radius coordinate, $r$, between the adjacent nodes, i.e., $\mathbf{N}=\left(N_{i}, N_{i+1}\right)=(1-\eta, \eta)$ in which $\eta=(r-$ $\left.r_{i}\right) / \Delta r, \Delta r=r_{i+1}-r_{i}$. Thus, the finite element equations from which Eq. 9 is assembled are as follows (2):

$\frac{\Delta r}{12}\left[\begin{array}{cc}4 r_{i}+\Delta r, & 2 r_{i}+\Delta r \\ 2 r_{i}+\Delta r, & 4 r_{i}+3 \Delta r\end{array}\right]\left\{\begin{array}{c}\dot{H}_{i}+i \omega H_{i} \\ \dot{H}_{i+1}+i \omega H_{i+1}\end{array}\right\}$

$+C(t) \frac{\bar{r}_{i}}{\Delta r}\left[\begin{array}{rr}1, & -1 \\ -1, & 1\end{array}\right]\left\{\begin{array}{c}H_{i} \\ H_{i+1}\end{array}\right\}=\left\{\begin{array}{l}0 \\ 0\end{array}\right\}$

in which $\bar{r}_{i}=\left(r_{i}+r_{i+1}\right) / 2$; and a superimposed dot denotes the time derivative. After assembling Eq. 9 from these equations, the last row must be replaced by the equation $H_{b}=1$ in which $H_{b}=$ value of $H$ at the node at the outer surface. 
The column matrix of stresses is $\sigma=\left(\sigma_{r}, \sigma_{\theta}, \sigma_{z}\right)^{T}$, and the strain-nodal displacement relation is introduced as $\epsilon=\left(\epsilon_{i}, \epsilon_{\theta}, \epsilon_{z}\right)^{T}=\mathbf{B}\left(u_{i}, u_{i+1}, u_{z}\right)$, in which the geometric matrix is

$\mathbf{B}=\left[\begin{array}{ccc}-(\Delta r)^{-1}, & (\Delta r)^{-1}, & 0 \\ -\left(2 \Delta \bar{r}_{i}\right)^{-1}, & \left(2 \bar{r}_{i}\right)^{-1}, & 0 \\ 0, & 0, & 1\end{array}\right]$

Here subscripts $r, \theta$, and $z$ refer to the components in the radial, circumferential, and axial directions; and $u_{i}$ are the radial nodal displacements. The cross sections are assumed to remain plane, and so the axial displacement, $u_{z}$, accumulated over a unit axial distance, is uniform throughout the cross section.

The finite element stiffness matrices from which Eq. 17 is assembled may then be calculated as $\mathbf{K}=\mathbf{B}^{T} \mathbf{D} \mathbf{B} \bar{r}_{i} \Delta r$, in which $\mathbf{D}$ is the elastic stiffness matrix of the material corresponding to Young's modulus, $E_{c}$, and Poisson ratio $\nu$. The column matrix of nodal forces equivalent to shrinkage strains is calculated as $\mathbf{F}_{s h} \simeq \mathbf{B}^{T} \mathbf{D} \epsilon_{s h} \bar{r}_{i} \Delta r$, in which $\epsilon_{s h}=$ $\left(\epsilon_{s h}, \epsilon_{s h}, \epsilon_{s h}, 0,0,0\right)^{T}$. The detailed forms of the matrices are

$K=\frac{E_{c}(1-v)}{(1+v)(1-2 v)}\left[\begin{array}{ccc}A_{1}, & A_{2}, & A_{3} \\ A_{2}, & A_{1}, & A_{4} \\ A_{3}, & A_{4}, & r_{i} \Delta r\end{array}\right], \quad \mathbf{F}_{s h}=\left[\begin{array}{c}-\sigma_{r}^{0} \bar{r}_{i}+\frac{1}{2} \sigma_{\theta}^{0} \Delta r \\ \sigma_{r}^{0} \bar{r}_{i}+\frac{1}{2} \sigma_{\theta}^{0} \Delta r \\ \sigma_{\theta}^{0} \bar{r}_{i} \Delta r\end{array}\right] \cdots$

in which $A_{1}=\left(\bar{r}_{i} / \Delta r\right)+\left(\Delta r / 4 \bar{r}_{i}\right)-v /(1-v) ; A_{2}=\left(\Delta r / 4 \bar{r}_{i}\right)-\left(\bar{r}_{i} / \Delta r\right) ; A_{3}$ $=-r_{i} \nu /(1-v) ; A_{4}=-r_{i+1} \nu /(1-v) ;$ and $\sigma_{r}^{0}, \sigma_{\theta}^{0}, \sigma_{z}^{0}=$ the components of the column matrix $\boldsymbol{\sigma}^{0}=\mathbf{D} \epsilon_{s h}$.

\section{Numerical Examples}

For illustration, two examples with different values of internal radius, $a$, and external radius $b$, are solved: (1) $a=20 \mathrm{~m}, b=21 \mathrm{~m}$; and (2) $=10 \mathrm{~m}, b=10.1 \mathrm{~m}$ (Fig. 1); in both examples, based on Wierig's data (25), $C(t)=\left(0.3+3.6 t^{-1 / 2}\right) 3 \mathrm{~cm}^{2} /$ day, in which $t$ must be given in days. The Poisson ratio is $v=0.18$, and the shrinkage coefficient is $\kappa_{s h}=0.0008$. The environmental relative humidity varies sinusoidally with the period $T=365$ days; $h=h_{m}+s_{0} \cos \omega\left(t-t_{0}\right)=0.7+0.2 \cos [2 \pi(t-28) /$ $365]$, i.e., the mean annual value of $h$ is 0.7 , its standard deviation is $s_{0}$ $=0.2$, its circular frequency is $\omega=2 \pi / 365$, and the age of concrete at the beginning of exposure to the environment is $t_{0}=28$ days. The initial pore humidity of concrete is $h_{0}=1$. However, for the purpose of calculating the frequency response, we use $h_{0}=0$ and $h=0.2 \exp (2 \pi i t)$ 365 ) at the surface. The surface humidity after exposure is assumed to be equal to the environmental humidity. Although it is no problem to run the computer program for an environmental humidity consisting of several periodic components, we present here only the solution for a single periodic component since it is more easily understood and interpreted.
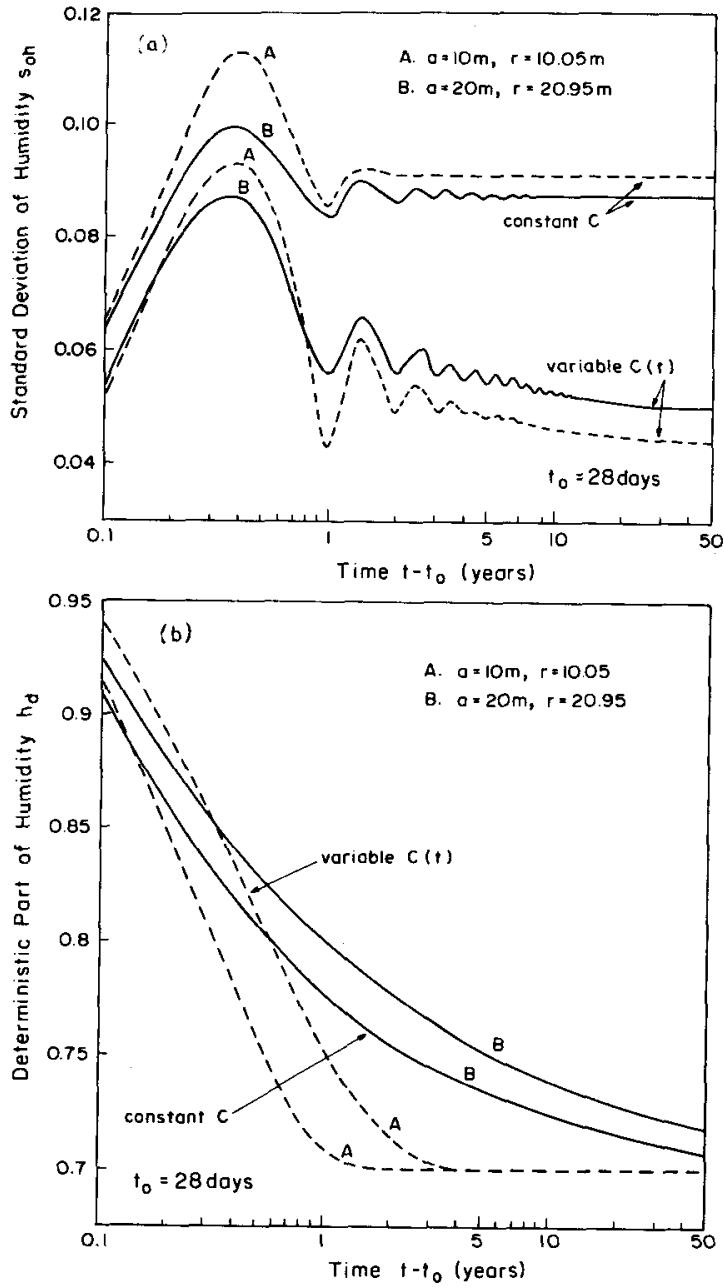

FIG. 2.- $(a-b)$ Time Evolution of Standard Deviation of Pore Humidity and of Its Deterministic Part (at $5 \mathrm{~cm}$ below Exposed Surface)

The relaxation function $R\left(t, t^{\prime}\right)$ of concrete is taken from Ref. 12 as determined from the test data for Ross Dam Concrete. The effect of pore humidity variation on the creep law is neglected; so is the tensile cracking due to shrinkage stresses. This means that the present calculation is valid only if the vessel is prestressed and the prestress is sufficient to make the maximum tensile stress less than the tensile strength of concrete. A computer program to implement the present solution was written in complex arithmetic. Two solutions were run: one for the mean environmental humidity, and one for its fluctuating component. In the former solution, the time step was increased in a geometric progression, while in the latter situation it could not be increased. Thus, the solution 


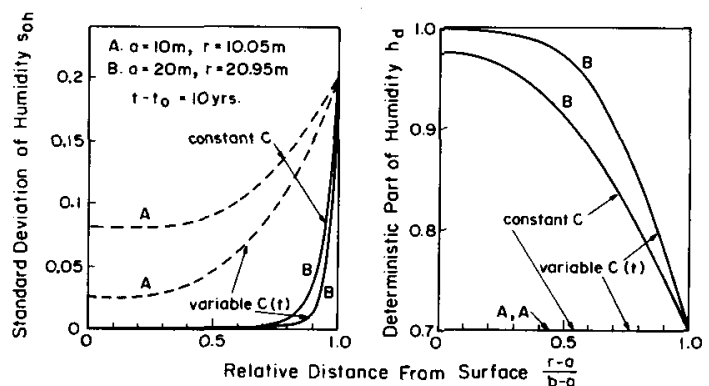

FIG. 3.-Profiles of Standard Deviation of Humidity and Its Deterministic Part at Age $10 \mathrm{Yr}$
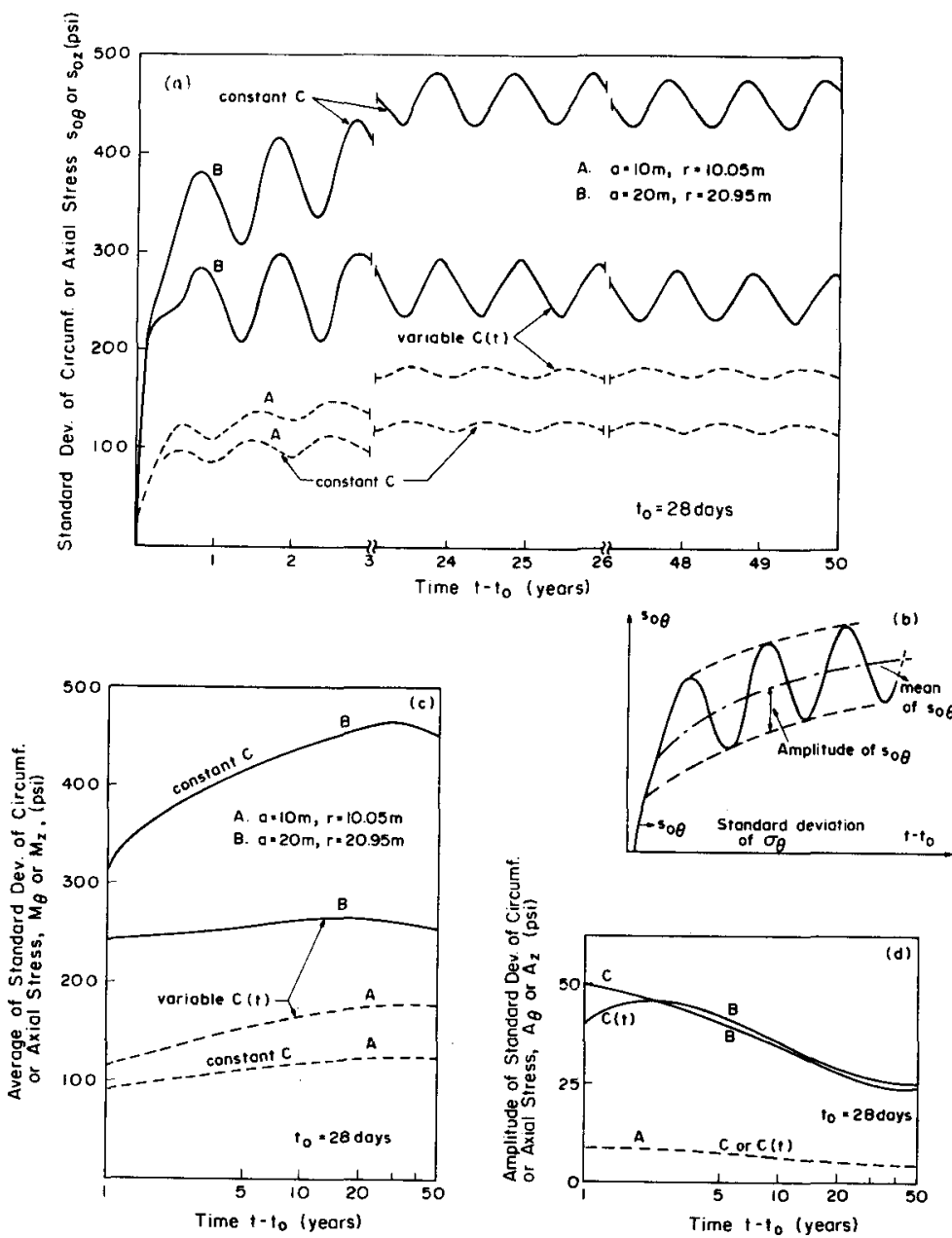

FIG. 4.-(a-d) Time Evolution of Standard Deviation of Clrcumferential or Axia Shrinkage Stresses, Mean of Standard Deviation, and Its Amplitude
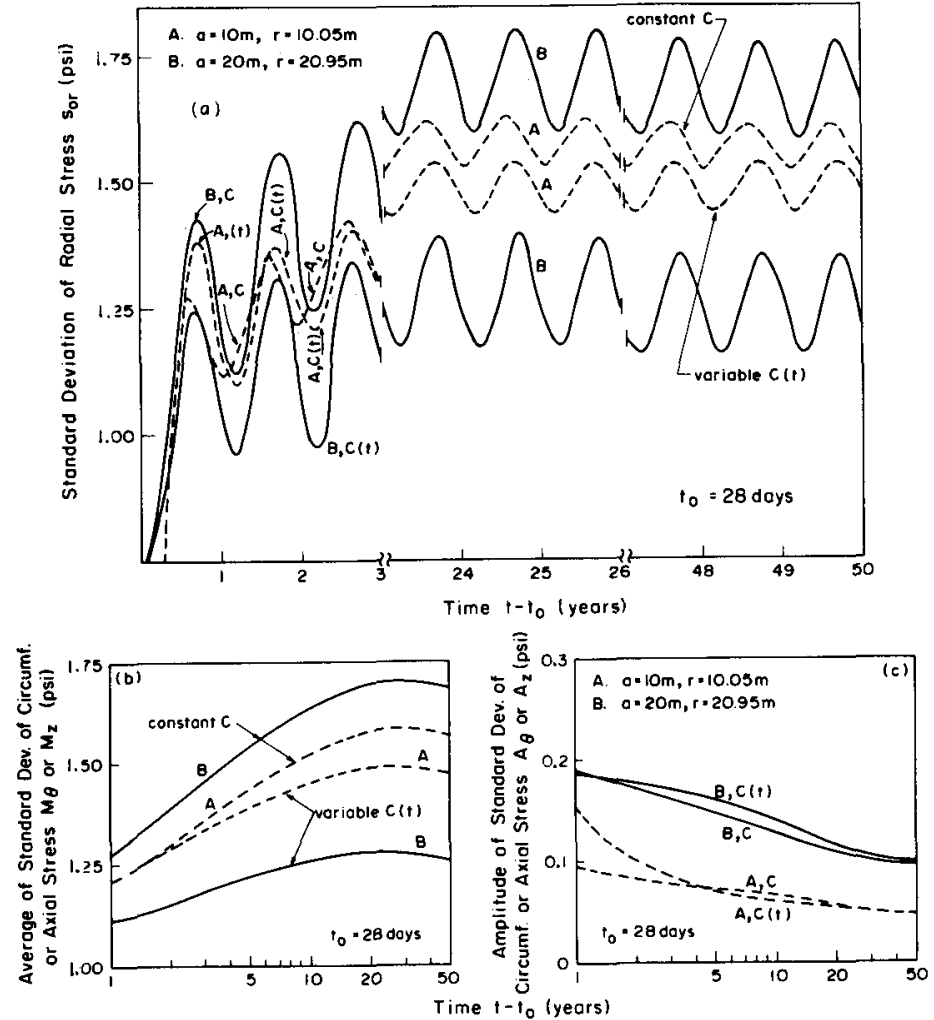

FIG. 5.-TIme Evolution of Standard Deviation of Radlal Shrinkage Stress, and of Its Mean and Amplitude (at $5 \mathrm{~cm}$ below Outer Surface)

of the fluctuating component required far more computing time. The number of time steps for the fluctuating component was $16\left(t_{n}-t_{0}\right) \omega /$ $2 \pi$ in which $t_{n}=$ the given final age of computation $(50 \mathrm{yr})$. The element subdivision of the wall, $1 \mathrm{~m}$ thick, consisted of 7 elements of $0.1 \mathrm{~m}, 5$ elements of $0.04 \mathrm{~m}$ and 5 elements of $0.02 \mathrm{~m}$.

The numerical results are plotted in Figs. 2-6. All plots of time variation are given for the points located at $5 \mathrm{~cm}$ from the outer surface. All the spatial distribution diagrams are plotted for the age $t=10 \mathrm{yr}$.

Fig. 2(a) shows that the history of standard deviation of pore humidity oscillates significantly up to about $t=10 \mathrm{yr}$. The solutions have been obtained both for the age-dependent humidity given above and for a constant diffusivity, $C$, which equals the value of $C(t)$ at 28 days. Fig. $2(b)$ shows that the decrease of diffusivity with age causes an increase in the mean level of humidity. The opposite happens with the standard deviation of humidity, $s_{0 h}$. While $s_{0 h}$ for the case of constant $C$ reaches a stationary value at the age of $10 \mathrm{yr}$, the plot of $s_{0 h}$ continues declining with age. The stresses are shown in Figs. 4-6. Because of the high value of ratio $a /(b-a)$, the values of $\sigma_{\theta}$ and $\sigma_{z}$ are so close that they cannot be distinguished graphically. 

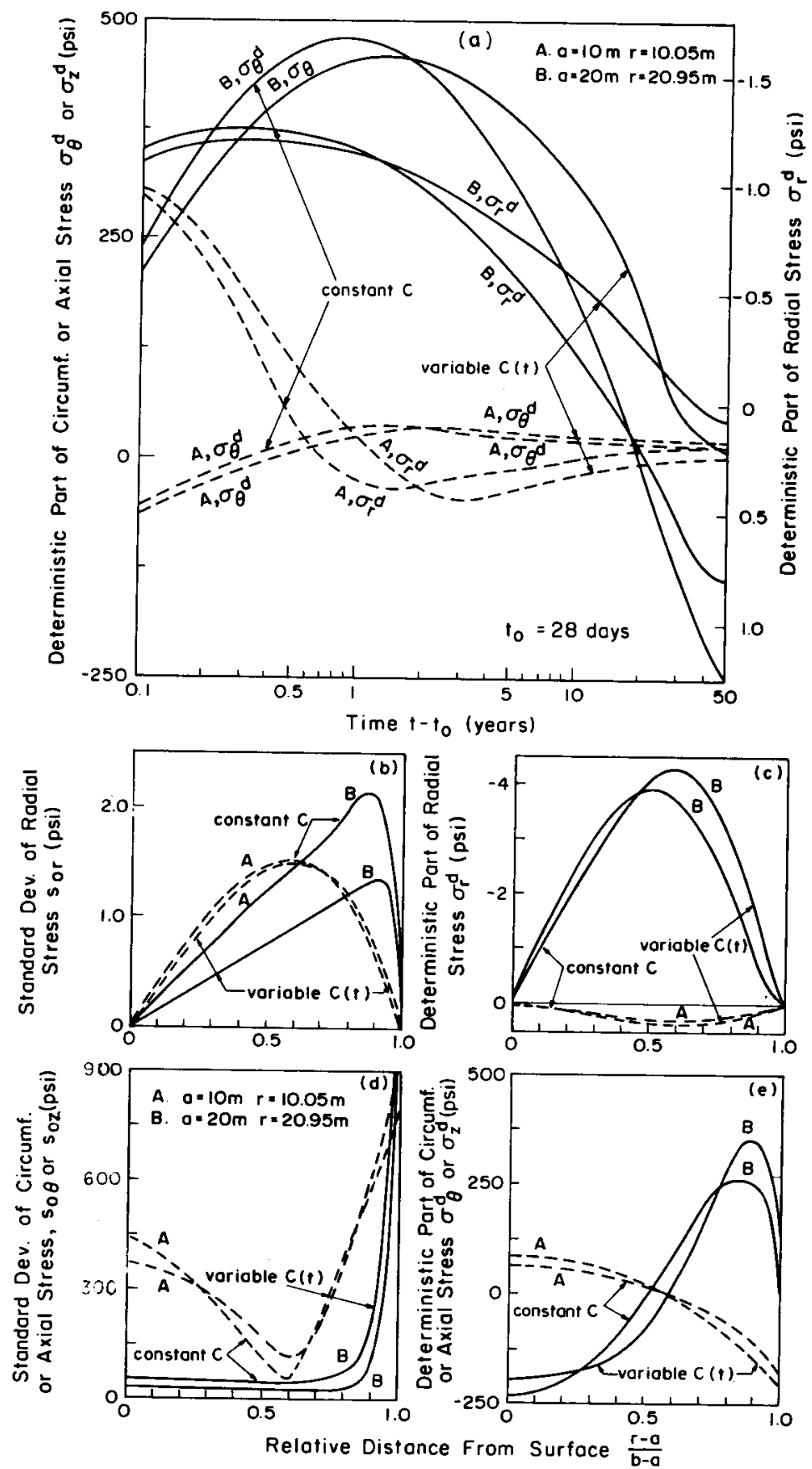

FIG. 6. - (a) Time Evolution of Deterministic Part of Radial and Circumferential (or Axial) Shrinkage Stresses; and (b-e) Profiles of Standard Deviation of Radlal and Circumferential (or Axial) Shrinkage Stresses and of Their Deterministlc Part
The time variations of the standard deviations of stresses, $s_{0 \sigma_{r}}, s_{0 \sigma_{\theta}}$, and $s_{\mathrm{O \sigma}_{2}}$, are shown in Figs. 4(a) and 5(a). It is noteworthy that they oscillate except for the first few months. The same observation has been made from the analytical solution of a halfspace in Ref. 11. The amplitudes $A_{r}, A_{\theta}, A_{z}$, and the averages $M_{r}, M_{\theta}, M_{z}$ of the time variation of these standard deviations of stresses, which were determined in the manner shown in Fig. $4(b)$, are plotted in Fig. $4(c-d)$, and Fig. $5(b-c)$. The values of these averages increase with time. Most of them reach their maximum between 20 and $30 \mathrm{yr}$, and then they slightly decrease. The amplitudes of the oscillations of the standard deviations of stresses monotonically decrease with time except for the first two years.

For constant diffusivity, $C$, the solution has been obtained in Ref. 24 with the help of Bessel and Kelvin functions. The fluctuations of the standard deviations of the stresses were, however, missed in Ref. 24, not because of the solution method, but because the values of the three standard deviations were evaluated only at points spaced too far apart in time.

\section{Conclusions}

1. The spectral method, which has been previously generalized for aging linear systems $(5,6)$, can be applied in conjunction with the finite element method to allow analysis of linearly viscoelastic structures of arbitrary geometry which undergo aging.

2 . The response stresses represent a random process that is nonstationary, for two reasons: (a) The initial condition; and (b) the age dependences of the drying diffusivity and of the creep properties. The effect of aging is very significant.

3. The standard deviation of the response humidity or shrinkage stress exhibits oscillations about a drifting mean.

4. Since the stresses are computed in the present method by a superposition integral based on the relaxation function, the time steps must be small compared to the shortest oscillation period involved. Thus, the computing time can be quite large when short periods are considered to be present in the environment. (For a more sophisticated method which avoids that see Ref. 10.)

\section{ACKNOWLEDGMENT}

Partial financial support under U.S. National Science Foundation grant No. CEE8303148 is gratefully acknowledged. Mary Hill is thanked for her outstanding secretarial assistance.

\section{Appendix.-References}

1. ASCE Structural Division Task Committee on Finite Element Analysis of Reinforced Concrete, "Finite Element Analysis of Reinforced Concrete (Stateof-the-Art Report)" ASCE, Chpt 6, N.Y., 1982, pp. 309-400.

2 Bazant $Z$ P "Numerical Analysis of Long-time Deformations of a Thickwalled Concrete Cylinder," Report No. 69-12, Structure and Materials Research, Civil Engineering Department, University of California, Berkeley, Calif., Aug., 1969. 
3. Bažant, Z. P., "Theory of Creep and Shrinkage in Concrete Structures: A Précis of Recent Developments," Mechanics Today, Vol. 2, Pergamon Press, New York, N.Y., 1975, pp. 1-93.

4. Bažant, Z. P., "Mathematical Models for Creep and Shrinkage of Concrete," Creep and Shrinkage in Concrete Structures, Z. P. Bažant and F. H. Wittmann, eds., J. Wiley, London, England, 1982.

5. Bažant, Z. P. " "Response of Aging Linear Systems to Random Input," Cente for Concrete and Geomaterials, Report No. 82-12/6654, Northwestern University, Evanston, Ill., Dec., 1982.

6. Bažant, Z. P., "Probabilistic Problems in Prediction of Creep and Shrinkage Effects in Structures," Proceedings of the 4th International Conference on Application of Statistics and Probability in Soil and Structural Engineering, G. Augusti, ed., Florence, Italy, June, 1983, pp. 325-356.

7. Bažant, Z. P., and Asghari, A., "Computation of Age-Dependent Relaxation Spectra," Cement \& Concrete Research, Vol. 4, 1974, pp. 567-579.

8. Bažant, Z. P. Carreira, D., and Walser, A., "Creep and Shrinkage in Reactor Containment Shells," Journal of the Structural Engineering Division, ASCE, Vol. 101, 1975, pp. 1997-2004.

9. Bažant, Z. P., and Najjar, L. J., "Nonlinear Water Diffusion in Nonsaturated Concrete," Materials and Structures, RILEM, Paris, Vol. 5, 1972, pp. 3-20.

10. Bažant, Z. P., and Wang, T.-S., "Algorithm for Aging Linear Systems under Periodic Loads," Report No. 83-9/679a, Center for Concrete and Geomaterials, Northwestern University, Evanston, Ill., 1983; also Journal of Engineering Mechanics, ASCE, Vol. 110, No. 6, June, 1984, pp. 972-984.

11. Bažant, Z. P., and Wang, T. S., "Spectral Analysis of Random Shrinkage in Concrete Structures," Journal of Engineering Mechanics, ASCE, Vol. 110, No. 2, Feb., 1984, pp. 173-186.

12. Bažant, Z. P., and Wu, S. T., "Creep and Shrinkage Law for Concrete at Variable Humidity," Journal of the Engineering Mechanics Division, ASCE, Vol. 100, No. EM6, Dec., 1974, pp. 1183-1207.

13. Bažant, Z. P., and Wu, S. T., "Rate-Type Creep Law of Aging Concrete Based on Maxwell Chain," Materials and Structures, Paris, France, Vol. 7, No. 37 1974, pp. 45-60.

14. Carslaw, H. S., and Jaeger, J. C., Conduction of Heat in Solids, 2nd ed., Clarendon Press, Oxford, 1959.

15. Crandall, S. H., Random Vibration in Mechanical Systems, Academic Press, New York, N.Y., 1963.

16. Heller, R. A., "Temperature Response of an Infinitely Thick Slab to Random Surface Temperatures," Mechanics Research Communications, Vol. 3, 1976, pp 379-385.

17. Heller, R. A., "Thermal Stress as a Narrow-Band Random Load," Journal of the Engineering Mechanics Division, ASCE, Vol. 102, No. EM5, Oct., 1976, pp. 787-805.

18. Heller, R. A., Kamat, M. P., and Singh, M. P., "Probability of Solid-Propellant Motor Failure Due to Environmental Temperatures," Journal of Spacecrafts and Rockets (AIAA), Vol. 20, 1983, pp. 144-149.

19. Heller, R. A., Kamat, M. P., and Sing, M. P., "Probability of Solid-Propellant Motor Failure Due to Environmental Temperatures," Journal of Spacecraft and Rockets (AIAA), Vol. 16, 1979, pp. 140-146.

20. Heller, R. A., Kamat, M. P., and Singh, M. P., "Probability of Solid-Propellant Motor Failure Due to Environmental Temperatures," Journal of Spacecrafts and Rockets, AIAA, 19th Joint Propulsion Conference, Seattle, 1984, Pre print No. AIAA-83-1120.

21. McHenry, D., "A New Aspect of Creep in Concrete and Its Application to Design," Proceedings, American Society for Testing and Materials, Vol. 43, 1943, pp. 1069-1086.

22. Papoulis, A., Probability, Random Variables and Stochastic Processes, McGrawHill, New York, N.Y., 1965.
23. Singh, M. P., and Heller, R. A. "Random Thermal Stresses in Nuclear Containments," Proceedings, ASCE Specialty Conference on Probabilistic Mechanics and Structural Reliability, A. H. S. Ang and M. Shinozuka, eds. Tucson, Ariz., Jan. 10-12, 1979, pp. 16-19.

24. Tsubaki, T., and Bažant, Z. P., "Random Shrinkage Stresses in Aging Viscoelastic Vessel," Joumal of the Engineering Mechanics Division, ASCE, Vol. 108, No. EM3, June, 1982, pp. 527-545.

25. Wementmörtel und Be . Wierig, H. J., "Die Wasserdam

26. Zienkiewicz, O.C., The Finite Element Method, 3rd Ed., 1977, McGraw-Hill New York, N.Y. 